\title{
DEVELOPMENT OF A NEW CONTINUOUS INTRAOPERATIVE NEUROMUSCULAR MONITORING SYSTEM USING PHONOMYOGRAPHY
}

Guillaume Trager, MSc, ${ }^{1,2}$ Stéphane Deschamps, MSc, ${ }^{1,2}$ Pierre A. Mathieu, $\mathrm{PhD},{ }^{2}$ Thomas Hemmerling, MD, DEAA $^{1}$

${ }^{1}$ Neuromuscular Research Group, Department of Anesthesiology, Centre Hospitalier de l'Université de Montréal, Université de Montréal and ${ }^{2}$ Institut de Génie Biomédical, Université de Montréal

INTRODUCTION. Phonomyography can be used interchangeably with mechanomyography for neuromuscular monitoring during anesthesia. The purpose of this work is to develop a monitoring system based on this novel method.

METHDOS. We developed a monitoring system which can record simultaneously the neuromuscular blockade of two muscles using interface designed using LABVIEW $^{\circledR}$ software. The aim was to display signals derived from two piezo-electric microphones and to analyse these signals in real time providing trends of two muscles. The system should offer neuromuscular monitoring with or without control stimulation and simple Train-offour-ratio (TOF) measurements. The occurrence rate of artefacts and the system reliability was tested in 10 patients undergoing general anesthesia. The workload caused by and performance of the interface was evaluated by 8 anesthesiologists using the NASA TLX ${ }^{\circledR}$-questionnaire.

RESULTS. The interface displays continuously acoustic raw signals for two different muscles (Fig. 1 a). The interface is easy to use with minimal physical, mental or temporal demands permitting high user performances after minimal training. It offers the option for data recording, reviewing or data transfer (Fig. 1 a). Additional features include simple artefact protection tools, such as the exclusion of electrocautery-induced artefacts by limiting the maximum amplitude recorded in the trend display. The performance of the monitoring system in relation to the time lost to artefacts was low at a mean of $10 \% \pm 2 \%$ of the monitoring time. Workload and physical or mental demands are low with high performance and low frustration levels. (Fig. $1 \mathrm{~b}$ )

CONCLUSION. A graphically oriented monitoring system based on phonomyography was created offering three modes of simultaneous monitoring of two muscles: monitoring with or without reference stimulation, and simple TOF-ratio measurements. The system is intuitive; resembling everyday life user interfaces, is easy-touse and provides artefact protection tools..

Figure $1 \mathrm{a}$

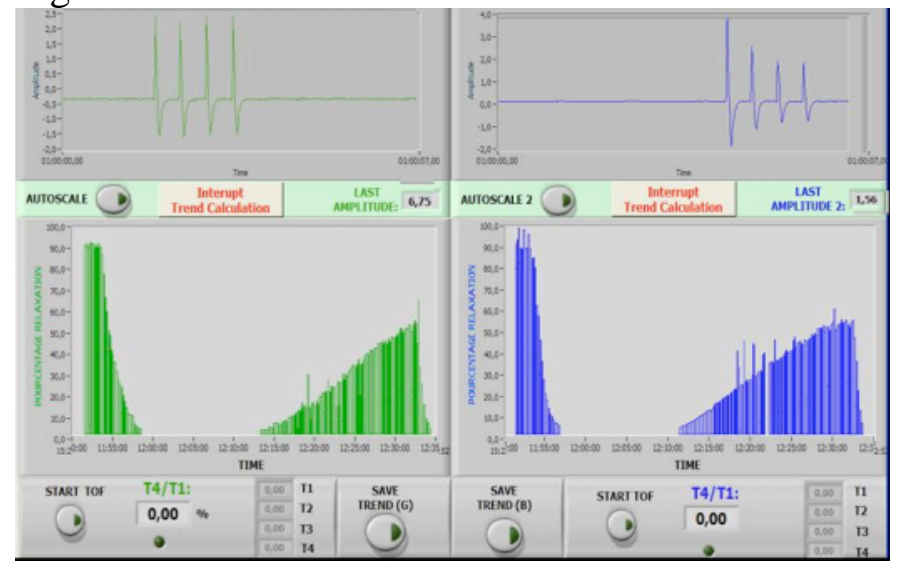

Figure $1 b$

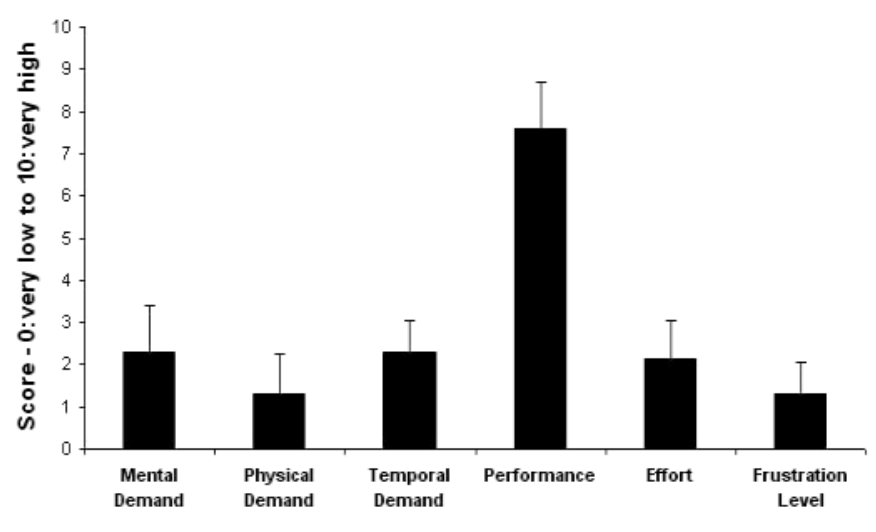

